\title{
Atomic and molecular adsorption on $\mathrm{Rh}(111)$
}

\author{
M. Mavrikakis, ${ }^{\text {a) }}$ J. Rempel, and J. Greeley \\ Department of Chemical Engineering, University of Wisconsin-Madison, Madison, Wisconsin 53706 \\ L. B. Hansen and J. K. Nørskov \\ Center for Atomic-scale Materials Physics, Department of Physics, Technical University of Denmark, \\ DK-2800 Lyngby, Denmark
}

(Received 10 January 2002; accepted 24 July 2002)

\begin{abstract}
A systematic study of the chemisorption of both atomic $(\mathrm{H}, \mathrm{O}, \mathrm{N}, \mathrm{S}, \mathrm{C})$, molecular $\left(\mathrm{N}_{2}, \mathrm{CO}, \mathrm{NO}\right)$, and radical $\left(\mathrm{CH}_{3}, \mathrm{OH}\right)$ species on $\mathrm{Rh}(111)$ has been performed. Self-consistent, periodic, density functional theory (DFT-GGA) calculations, using both PW91 and RPBE functionals, have been employed to determine preferred binding sites, detailed chemisorption structures, binding energies, and the effects of surface relaxation for each one of the considered species at a surface coverage of $0.25 \mathrm{ML}$. The thermochemical results indicate the following order in the binding energies from the least to the most strongly bound: $\mathrm{N}_{2}<\mathrm{CH}_{3}<\mathrm{CO}<\mathrm{NO}<\mathrm{H}<\mathrm{OH}<\mathrm{O}<\mathrm{N}<\mathrm{S}<\mathrm{C}$. A preference for threefold sites for the atomic adsorbates is observed. Molecular adsorbates, in contrast, favor top sites with the exceptions of $\mathrm{NO}$ (hcp) and $\mathrm{OH}$ (fcc or bridge tilted). Surface relaxation leads to insignificant changes in binding energies but to considerable changes in the spacing between surface rhodium atoms, particularly for on-top adsorption where the rhodium atom directly below the adsorbate is lifted above the plane of the surface. RPBE binding energies are found to be in remarkable agreement with the available experimental values. All atomic adsorbates, except for $\mathrm{H}$, have a significant diffusion barrier [between 0.4 and $0.6 \mathrm{eV}$ (RPBE)] on $\mathrm{Rh}(111)$. Atomic $\mathrm{H}$ and molecular/radical adsorbates appear to be much more mobile on $\mathrm{Rh}(111)$, with an estimated diffusion barrier between 0.1 and $0.2 \mathrm{eV}$ (RPBE). Finally, the thermochemistry for dissociation of $\mathrm{CO}, \mathrm{NO}$, and $\mathrm{N}_{2}$ on $\mathrm{Rh}(111)$ has been examined. In all three cases, decomposition is found to be thermodynamically preferable to desorption. (C) 2002 American Institute of Physics.
\end{abstract}

[DOI: $10.1063 / 1.1507104]$

\section{INTRODUCTION}

Rhodium is an extremely valuable and versatile transition metal for applications in heterogeneous catalysis. For example, current catalytic converter technology employs the three-way-catalyst (TWC). The TWC, made of supported $\mathrm{Pt}-\mathrm{Rh}$, can catalyze the simultaneous conversion of nitric oxide, carbon monoxide, and unburned hydrocarbons. ${ }^{1}$ Rhodium can also catalyze the hydroformylation reaction (aldehyde production from olefins, carbon monoxide, and hydrogen) and the catalytic partial oxidation of methane. ${ }^{1}$

Natural reserves of rhodium are currently in rapid decline, and as a result $\mathrm{Rh}$ is by far the most expensive precious metal today. Hence, it is of great importance to find new catalysts with high activity and selectivity to replace the current generation of rhodium catalysts. Understanding the surface chemistry of rhodium might suggest efficient ways of proceeding with this catalyst design process. In particular, understanding critical elementary reaction steps such as adsorption and bond breaking/making over rhodium surfaces can provide substantial insights into why rhodium is so essential in catalysis and as to how it can best be replaced.

The interaction of atomic species with rhodium single crystal surfaces has been the subject of several research stud-

\footnotetext{
a) Author to whom correspondence should be addressed. Electronic mail: manos@engr.wisc.edu; Phone: (608) 262-9053; Fax: (608) 262-5434.
}

ies in the past. Adsorbed oxygen atoms on $\mathrm{Rh}(111)$ have been investigated with low-energy electron diffraction (LEED) (Refs. 2 and 3) and with molecular-beam scattering experiments. ${ }^{4}$ The overlayer structure of adsorbed atomic nitrogen on $\mathrm{Rh}(111)$ was studied with scanning tunneling microscopy (STM). ${ }^{5}$ For a detailed review of $\mathrm{O}$ and $\mathrm{N}$ chemistry on $\mathrm{Rh}(111)$ and other single-crystal rhodium facets, see Comelli et al. ${ }^{6}$ The structures formed by adsorbed sulfur on $\mathrm{Rh}(111)$ have been studied by LEED (Refs. 7 and 8) and STM (Ref. 8) experiments. Finally, high resolution electron energy loss spectra (HREELS) and laser-induced thermal desorption studies have been carried out on $\mathrm{Rh}(111)$ to study hydrogen adsorption ${ }^{9}$ and hydrogen diffusion, ${ }^{10}$ respectively.

Experimental studies of molecular adsorption on $\mathrm{Rh}(111)$ are even more abundant. For example, using temperature programmed desorption (TPD), Hendrickx et al. examined the adsorbed states of dinitrogen $\left(\mathrm{N}_{2}\right)$ on rhodium (111), (100), (110), and (210) surfaces. ${ }^{11}$ LEED, EELS, and TPD studies have been carried out to study the chemisorption of nitric oxide (NO) on $\mathrm{Rh}(111)$ and $\mathrm{Rh}(100))^{12-14}$ LEED has also been used to study coadsorbed overlayers of $\mathrm{NO}$ and $\mathrm{O}$ on $\mathrm{Rh}(111) .^{5}$ The adsorption and energetics of carbon monoxide $(\mathrm{CO})$ have been the subject of several experimental studies. LEED experiments have been used to determine site preferences for $\mathrm{CO}$ on $\mathrm{Rh}(111)$ at both low and high coverages. ${ }^{15-17}$ Numerous overlayer structures for $\mathrm{CO}$ on $\mathrm{Rh}(111)$ have also been observed with LEED, ${ }^{12,15}$ 
high resolution photoemission (HRP), ${ }^{17}$ and STM (Ref. 18) experiments. Coadsorbed systems of $\mathrm{CO}$ and $\mathrm{O}$ have been considered in other LEED (Refs. 2 and 3) studies. Finally, reflection adsorption infrared spectroscopy (RAIRS) and TPD have been used to study the adsorption of the methyl radical $\left(\mathrm{CH}_{3}\right)$ on $\mathrm{Rh}(111) .{ }^{19}$

A substantial amount of experimental research has been done to gain understanding of the kinetics of adsorption, desorption, and reactions on rhodium surfaces. Borg et al. determined several kinetic parameters involved in nitric oxide decomposition on $\mathrm{Rh}(111)$ from TPD experiments. ${ }^{20} \mathrm{Tem}-$ perature programmed reaction spectroscopy (TPRS) has been employed to study carbon monoxide oxidation on $\mathrm{Rh}(100)$ and $\mathrm{Rh}(111) .{ }^{21}$ The kinetics of the simultaneous reaction $\mathrm{NO}+\mathrm{CO}$ have also been widely studied using TPD (Ref. 22) and TPRS (Ref. 23) techniques on Rh(111). Finally, TPD has been used to analyze the recombination of atomic nitrogen on $\mathrm{Rh}(111)$ at high surface coverages. ${ }^{24}$

Several theoretical studies have been performed to study chemisorption and reactivity on rhodium surfaces. Periodic DFT calculations have been used to investigate atomic oxygen binding on $\mathrm{Rh}(111),{ }^{25-27}$ and the adsorption of other atomic species such as nitrogen, ${ }^{26}$ sulfur, ${ }^{28}$ and hydrogen ${ }^{27}$ has also been considered on this surface. Zhang et al. carried out periodic DFT studies to determine interactions between chemisorbed $\mathrm{CO}$ and $\mathrm{S}$ on $\mathrm{Rh}(111){ }^{28}$ General trends in the dissociation of $\mathrm{CO}$ have been studied on the (111) surfaces of $\mathrm{Rh}, \mathrm{Ru}, \mathrm{Pd}$, Os, Ir, and Pt, yielding the corresponding potential energy surfaces. ${ }^{29,30}$ Loffreda et al. performed periodic DFT calculations on rhodium (100) and (111) surfaces for molecular and dissociative chemisorption of nitric oxide. ${ }^{26}$ Water formation on $\mathrm{Rh}(111)$ surfaces has also been explored using DFT, providing thermodynamic and kinetic parameters. ${ }^{27}$

In the present work we present a systematic survey of the chemisorption structures and energetics of several atomic $(\mathrm{H}, \mathrm{O}, \mathrm{N}, \mathrm{S}, \mathrm{C})$, molecular $\left(\mathrm{N}_{2}, \mathrm{CO}, \mathrm{NO}\right)$, and radical $\left(\mathrm{CH}_{3}, \mathrm{OH}\right)$ species, all of which play a role in industrial rhodium-catalyzed reactions, on the $\mathrm{Rh}(111)$ surface. We use periodic, self-consistent DFT calculations to determine preferred binding sites and binding energies for the adsorbed species. We also estimate diffusion barriers and examine the thermochemistry of $\mathrm{CO}, \mathrm{NO}$, and $\mathrm{N}_{2}$ dissociation on $\mathrm{Rh}(111)$.

\section{METHODS}

All calculations are carried out using DACAPO ${ }^{31}$ A threelayer slab of rhodium, periodically repeated in a super cell geometry with five equivalent layers of vacuum between any two successive metal slabs, is used. A $2 \times 2$ unit cell, corresponding to a surface coverage of 0.25 ML, is used. Adsorption is allowed on only one of the two exposed surfaces, and the electrostatic potential is adjusted accordingly. ${ }^{32}$ Initial computations are performed with metal atoms fixed in their bulk-truncated positions. The calculations are then repeated for all geometries allowing the top metal layer to relax. Ionic cores are described by ultrasoft pseudopotentials,${ }^{33}$ and the Kohn-Sham one-electron valence states are expanded in a basis of plane waves with kinetic energy below $25 \mathrm{Ry}$. The
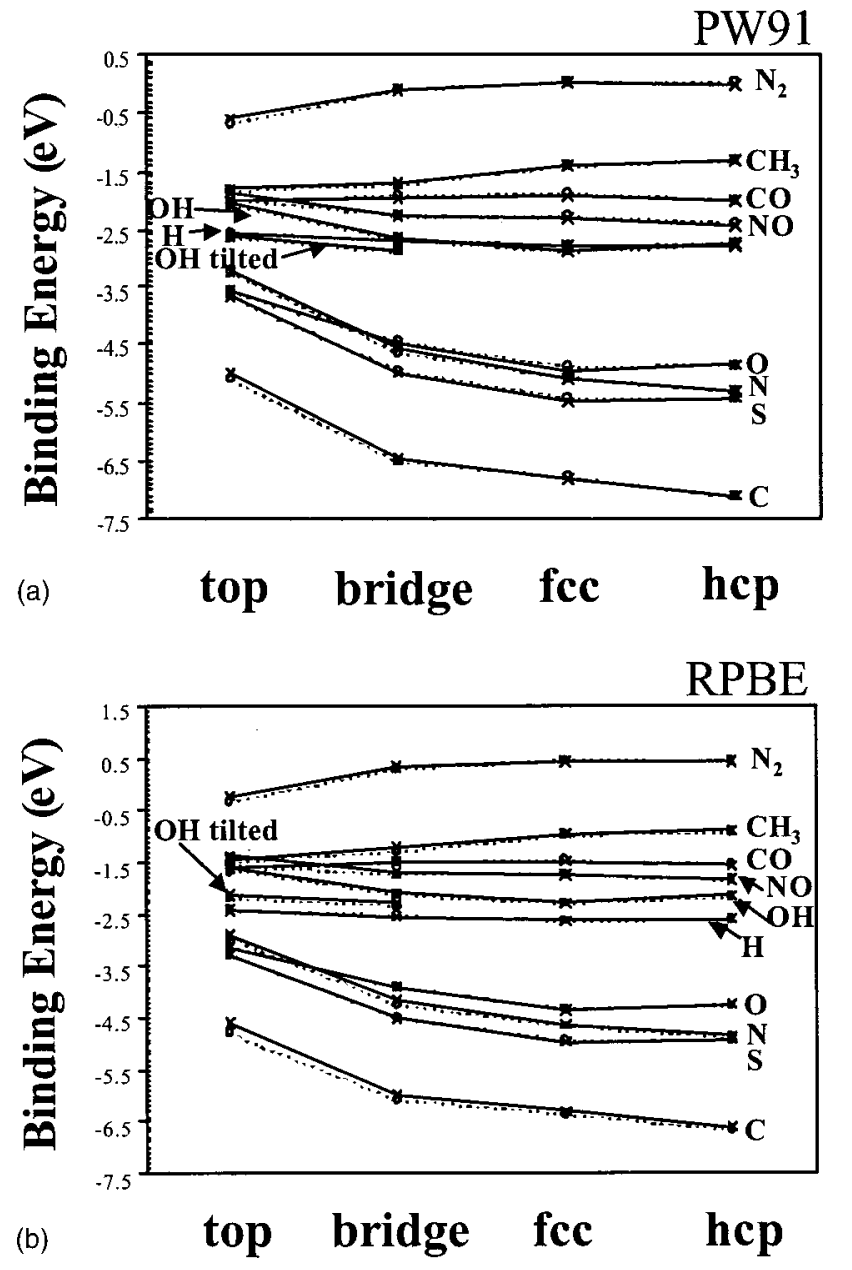

FIG. 1. Binding energies of atomic, molecular, and radical adsorbates on $\mathrm{Rh}$ (111), calculated with the (a) PW91 and (b) RPBE functionals (solid line, fixed surface; dashed line, relaxed surface). Reference zero corresponds to gas phase species at infinite separation from the surface. Lines are only guides to the eye.

surface Brillouin zone is sampled at 18 special $\mathbf{k}$ points. In all cases, convergence with respect to the $\mathbf{k}$ point set and with respect to the number of metal layers included is confirmed. The exchange-correlation energy and potential are described by two generalized gradient approximations, selfconsistently with GGA-PW91 (Refs. 34 and 35) and nonself-consistently with RPBE. ${ }^{31}$ The RPBE functional has been shown to give better chemisorption energies compared to PW91. ${ }^{31}$ The self-consistent PW91 density is determined by iterative diagonalization of the Kohn-Sham Hamiltonian, Fermi population of the Kohn-Sham states $\left(k_{B} T=0.1 \mathrm{eV}\right)$, and Pulay mixing of the resulting electronic density. ${ }^{36}$ All total energies have been extrapolated to $k_{B} T=0 \mathrm{eV}$. The calculated lattice constant for bulk rhodium, $3.83 \AA$, is within $1 \%$ of the experimental value of $3.797 \AA .{ }^{37}$

\section{RESULTS AND DISCUSSION}

This section contains a description of the chemisorption properties for all the adsorbates studied, including binding energies, diffusion barrier estimates, site preferences, and the effect of surface relaxation. Binding energies are presented for both PW91 and RPBE functionals, with the latter given 
TABLE I. Binding energies of atomic adsorbates on a relaxed $\mathrm{Rh}(111)$ surface. Preferred sites are shown by shaded entries. The reference zero corresponds to gas-phase atoms at infinite separation from the rhodium slab. (Experimental estimates are obtained from the gas-phase diatomic bond energy. ${ }^{\mathrm{b}, \mathrm{c}}$ )

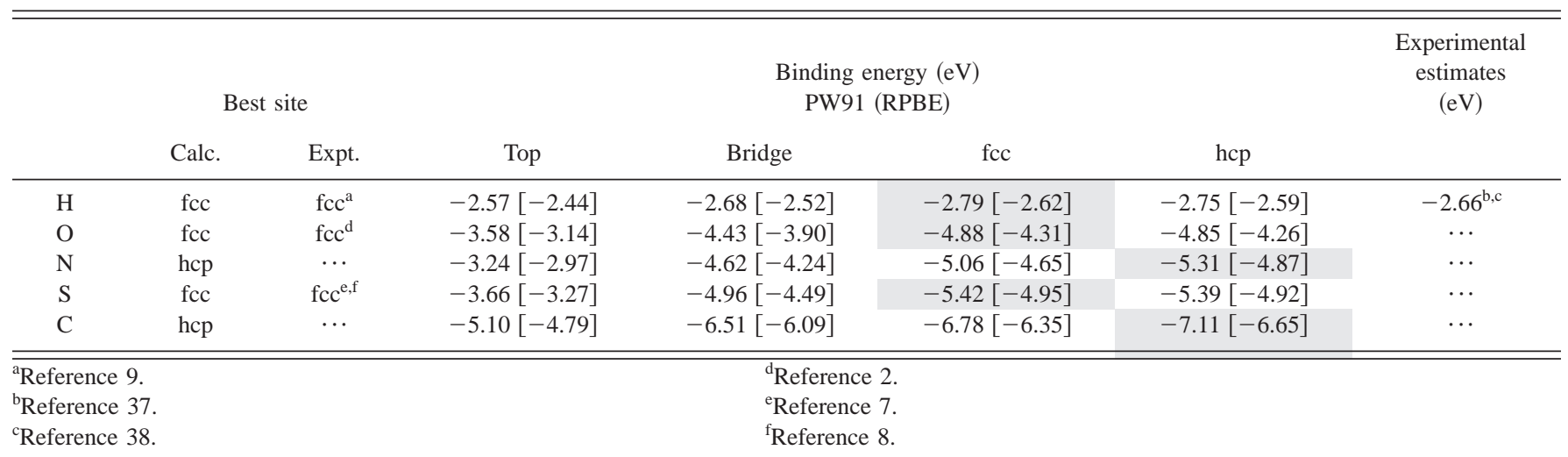

in square brackets. The effect of surface relaxation on the binding energies and that of coordination numbers on the binding site preferences are discussed. A summary of the calculated binding energies for fixed and relaxed surfaces for atomic, molecular, and radical adsorbates is presented in Fig. 1. The thermochemistry of adsorption and decomposition of carbon monoxide, nitric oxide, and dinitrogen is then presented. All results are compared to available experimental and theoretical data from the literature.

\section{A. Adsorption of atoms}

Atomic hydrogen is the least strongly bound atom among those we studied on $\mathrm{Rh}(111)$, with a binding energy of -2.79 [-2.62] eV (Table I). An experimental value for the binding energy can be estimated by combining the energy of dissociative adsorption of $\mathrm{H}_{2}$ on $\mathrm{Rh}(111),-0.807 \mathrm{eV}$ (obtained from TPD experiments ${ }^{38}$ ), with the $\mathrm{H}_{2}$ gas-phase bond enthalpy, $-4.52 \mathrm{eV}^{37}$ These values yield an estimated atomic hydrogen binding energy of $-2.66 \mathrm{eV}$, in excellent agreement with the RPBE value. In addition, a previous calculation indicates a binding energy of $-2.82 \mathrm{eV},{ }^{27}$ consistent with our findings. The most energetically favorable configuration in our calculations is the fcc site with the hydrogen atom very close to the surface. The geometric parameters for this configuration are presented in Table II, while Fig. 2 shows a schematic representation of the corresponding structural parameters. This site preference is consistent with HREELS experiments ${ }^{9}$ and with the theoretical study mentioned above. ${ }^{27}$ The hydrogen potential energy surface is

TABLE II. Vertical distance between the adsorbate and the plane of three metal atoms defining the corresponding site on relaxes surfaces $\left(d_{\mathrm{A}-\mathrm{Rh}}\right)$, displacement of the plane of three metal atoms defining the corresponding sites with respect to the clean, relaxed surface $\left(\Delta z_{\mathrm{Rh}}\right)$, and metal-metal bond length $\left(d_{\mathrm{Rh}-\mathrm{Rh}}\right)$ on the relaxed surface. A schematic representation is given in Fig. 2(a). $d_{\mathrm{Rh}-\mathrm{Rh}}$ on a clean surface $=2.71 \AA$.

\begin{tabular}{cccc}
\hline \hline Adsorbate & $\begin{array}{c}d_{\mathrm{A}-\mathrm{Rh}} \\
(\AA)\end{array}$ & $\begin{array}{c}\Delta z_{\mathrm{Rh}} \\
(\AA)\end{array}$ & $\begin{array}{c}d_{\mathrm{Rh}-\mathrm{Rh}} \\
(\AA)\end{array}$ \\
\hline H (fcc) & 0.98 & 0.02 & 2.74 \\
$\mathrm{O}$ (fcc) & 1.23 & 0.08 & 2.75 \\
$\mathrm{~N}$ (hcp) & 1.03 & 0.09 & 2.64 \\
$\mathrm{~S}$ (fcc) & 1.66 & 0.06 & 2.74 \\
$\mathrm{C}$ (hcp) & 1.00 & 0.09 & 2.62 \\
\hline \hline
\end{tabular}

found to be relatively flat in our calculations; an estimate for the H-diffusion barrier is 0.10 [0.10] eV (Table III). These findings are in close agreement with laser-induced thermal desorption experiments that indicate a diffusion barrier of $0.14 \mathrm{eV}$ for a $0.3 \mathrm{ML}$ coverage. ${ }^{10}$ Finally, the effect of surface relaxation is found to be very small in terms of energetics (0.02 eV destabilization).

The next least strongly bound atom is oxygen. The preferred binding site is fcc with a binding energy of -4.88 $[-4.31] \mathrm{eV}$ (Table I), and the estimated diffusion barrier is $0.45[0.41] \mathrm{eV}$ (Table III). The geometric parameters for the fcc site are presented in Table II. The preference for the fcc site is in agreement with LEED experimental findings ${ }^{2}$ and with previous DFT calculations. ${ }^{25}$ However, we find that the fcc site is preferred by only $0.03 \mathrm{eV}$ over the hcp site. This very small difference (discussed further below) raises the question of whether or not the site preferences could be significantly altered by zero-point energy effects; to assess the magnitude of such effects, we calculated the three normal vibrational modes and frequencies for oxygen in both the fcc and hcp sites. The total zero-point energy (including all three normal modes) of oxygen in the hcp site is roughly $100 \mathrm{~cm}^{-1}$ $(\sim 0.01 \mathrm{eV})$ greater than the corresponding energy in the fcc site, suggesting that zero-point effects are unlikely to alter site preferences for this system. In any case, at high surface

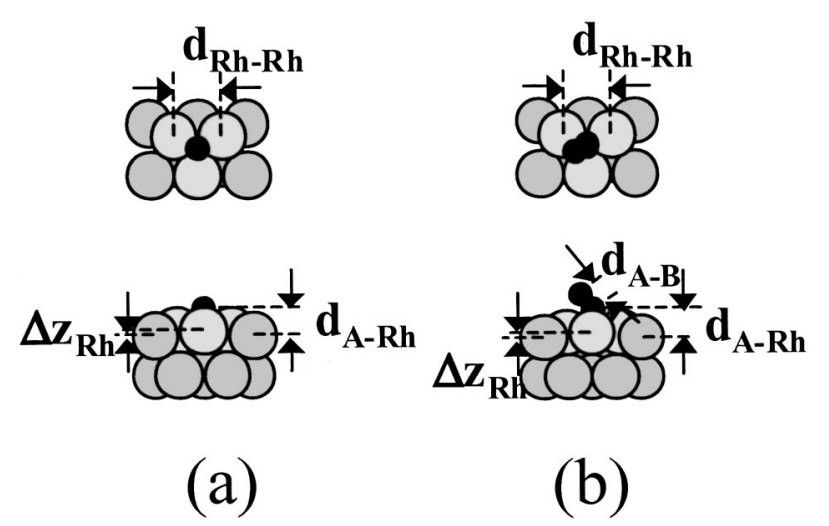

FIG. 2. Schematic representation of top and side views of the atomic and molecular adsorbates on threefold sites of Rh(111). (a) Atomic adsorbates. (b) Molecular and radical adsorbates. Geometrical parameters shown are used in Tables II and V. 
TABLE III. Estimates for the diffusion barriers of atomic species on $\mathrm{Rh}(111)$.

\begin{tabular}{ccc}
\hline \hline Adsorbate & $\begin{array}{c}\text { Diffusion barrier (PW91) } \\
(\mathrm{eV})\end{array}$ & $\begin{array}{c}\text { Diffusion barrier (RPBE) } \\
(\mathrm{eV})\end{array}$ \\
\hline $\mathrm{H}$ & 0.10 & 0.10 \\
$\mathrm{C}$ & 0.60 & 0.56 \\
$\mathrm{~N}$ & 0.68 & 0.64 \\
$\mathrm{O}$ & 0.45 & 0.41 \\
$\mathrm{~S}$ & 0.46 & 0.46 \\
\hline
\end{tabular}

coverages, population of either one of these sites can be observed. An experimental estimate of the binding energy can be obtained by combining the dissociative adsorption energy of molecular oxygen on $\mathrm{Rh}(100),-4.00 \mathrm{eV}$, obtained from femtomole adsorption calorimetry experiments, ${ }^{39}$ and the gas-phase $\mathrm{O}_{2}$ bond enthalpy, $-5.17 \mathrm{eV} .{ }^{37}$ These values give an estimated oxygen binding energy of $-4.58 \mathrm{eV}$ on $\mathrm{Rh}(100)$, which is reasonably close to our findings on $\mathrm{Rh}(111)$. Interestingly, there is quite a bit of controversy about the magnitude of the $\mathrm{O}$ binding energy in previous DFT calculations; values of $-5.03 \mathrm{eV}^{26}-5.22 \mathrm{eV},{ }^{25}$ and $-5.51 \mathrm{eV}$ (Ref. 27) have been reported. As with atomic hydrogen, the effect of surface relaxation on the oxygen binding energy is small. The vertical displacement of the coordinated rhodium atom is found to be $0.08 \AA$ (Table II), consistent with a $0.06 \pm 0.05 \AA$ experimental value. ${ }^{2}$ The oxygen to rhodium vertical distance, $1.23 \AA$ (Table II), is also in agreement with LEED experimental findings of 1.24 $\pm 0.06 \AA$. $^{2}$

The favored binding site for atomic nitrogen is hep with a binding energy of $-5.31[-4.87] \mathrm{eV}$ (Table I). This site preference does not agree with previous DFT calculations $(\theta=0.25 \mathrm{ML})$ that predict that the most stable site is fcc with a binding energy of $-4.55 \mathrm{eV} .{ }^{26}$ This discrepancy might be explained by differences between that calculation and ours; the functionals (Becke 88/Perdew 86 versus PW91), the treatment of core electrons (nonrelativistic frozen-cores versus relativistically corrected pseudopotentials), and the approximations for surface dynamics (no surface relaxation versus one surface layer relaxed) are different between the two calculations. In addition, it is worth noting that DFT calculations can sometimes lead to incorrect site preferences for small adsorbates. This problem has been analyzed in detail for the case of $\mathrm{CO} ;{ }^{40}$ a similarly extensive study would probably be needed to sort out the site preference discrepancies in the present case, and such a study is beyond the scope of this work. In any case, our computations yield a $0.25 \mathrm{eV}$ preference of the hcp site over the fcc site. The calculated estimate for the diffusion barrier of atomic nitrogen is 0.68 [0.64] eV (Table III). Surface relaxation yields an insignificant change in binding energy and a small vertical displacement of the rhodium atoms (Table II).

Even stronger bonding is exhibited by sulfur in fcc sites with a binding energy of $-5.42[-4.95] \mathrm{eV}$ (Table I). As in the case of atomic oxygen, sulfur has only a very weak preference $(\approx 0.03 \mathrm{eV})$ for fcc over hcp sites. The estimated diffusion barrier is 0.46 [0.46] eV (Table III). LEED experiments confirm that for coverages below 0.33 ML, sulfur occupies fcc sites. ${ }^{7,8}$ Previously reported DFT calculations yield a binding energy of $-5.25 \mathrm{eV}$ (fcc adsorption), ${ }^{28}$ in reasonable agreement with the present findings. For the adsorption in the fcc site, the change in binding energy due to surface relaxation is small. The geometric parameters of the preferred site are given in Table II.

The most strongly bound atom studied is carbon. The preferred binding site is hcp with a binding energy of -7.11 [-6.65] eV (Table I). The binding energy of the next most stable site, hcp, is $0.33 \mathrm{eV}$ higher in energy. The barrier for the diffusion of $\mathrm{C}$ is estimated to be 0.60 [0.56] eV (Table III). The change in binding energy due to surface relaxation is negligible. The geometric parameters of the preferred site are given in Table II.

For three of the atomic adsorbates discussed above $(\mathrm{H}$, $\mathrm{O}$, and $\mathrm{S}$ ), the magnitudes of the calculated binding energies (B.E.'s) for the hcp sites (the second most favorable sites in each case) are only $30-40 \mathrm{meV}$ less than the corresponding B.E.'s at the most favorable fcc sites. While we cannot say with certainty that these differences are numerically significant, the result that zero-point energy differences do not affect the site preferences (in the case of oxygen, at least), and the fact that our findings agree well with experimental results, allow us to be reasonably confident about our calculated site preferences. Furthermore, we note that our data support the explanation proposed by Ganduglia-Pirovano and Scheffler ${ }^{25}$ for the preference of oxygen for fcc sites. Those authors note that the workfunction of the rhodium surface decreases when adsorbed oxygen moves from the fcc to the hop site (we calculate $\Delta \phi=-0.14 \mathrm{eV}$ ). At the same time, the oxygen $s$-orbital energy decreases (we calculate $\Delta \epsilon_{s}=-0.17 \mathrm{eV}$ ). Both of these observations suggest that more charge is transferred from the surface to the oxygen atom in the fcc site, thereby leading to a more ionic bond with the surface and to a higher binding energy.

For the investigated atomic adsorbates a consistent preference for threefold sites is observed. This result indicates that gas-phase bonding trends are not always followed by atomic adsorbates on $\mathrm{Rh}(111)$. For example, oxygen and sulfur bind to at most two atoms in the gas phase while both adsorb in fcc sites on $\mathrm{Rh}(111)$. Similarly, hydrogen only binds to one atom in the gas phase, yet it prefers fcc sites on the rhodium surface. Nitrogen typically forms three bonds in the gas phase (although four bonds can be observed in species like ammonium), but it prefers hcp sites with four neighboring atoms on $\mathrm{Rh}(111)$. In the case of carbon, however, the gas phase tetravalent coordination is consistent with its preference for the hcp sites.

A general trend is observed in the geometry changes of top-layer rhodium atoms during surface relaxation for the fcc and hcp sites. For species adsorbed at $f c c$ sites $(\mathrm{H}, \mathrm{O}, \mathrm{S})$, Table II shows that there is an increase in $d_{\mathrm{Rh}-\mathrm{Rh}}$ from the equilibrium value. In other words, movement of rhodium atoms directed radially outward is observed for the fcc site. This trend has been observed in LEED experiments of adsorbed $\mathrm{O}$ on $\mathrm{Rh}(111) .^{2}$ On the other hand, for adsorbates at $h c p$ sites $(\mathrm{N}, \mathrm{C})$, the corresponding $d_{\mathrm{Rh}-\mathrm{Rh}}$ decreases from the equilibrium value. In this case there is movement of 
TABLE IV. Binding energies of molecular and radical adsorbates on a relaxed Rh(111) surface. All configurations are perpendicular to the surface, unless indicated otherwise. Best sites are shown by shaded entries. Reference zero corresponds to gas phase species at infinite separation from the rhodium slab. $\mathrm{NS}=$ not stable.

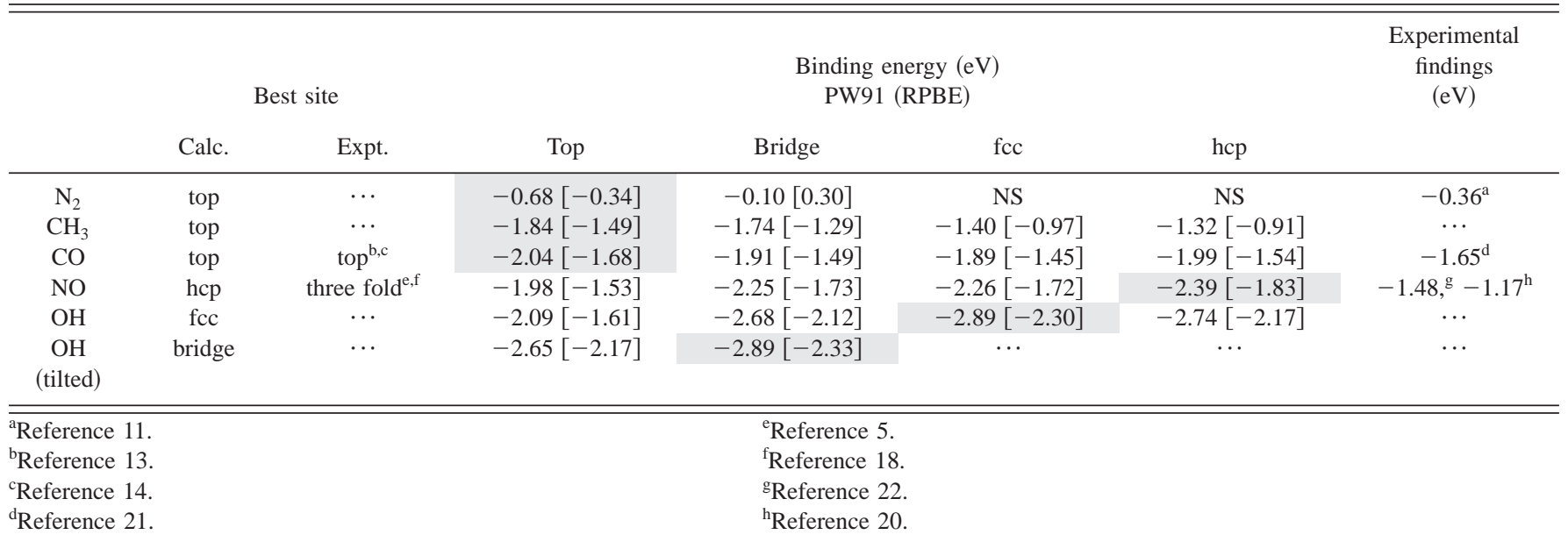

rhodium atoms directed radially inward. We could not find experimental data for comparison with these results.

For three of the above atomic adsorbates $(\mathrm{H}, \mathrm{O}$, and $\mathrm{S}$ ), the energetic preference for the most favored site (fcc, in these cases) is very weak $(<0.05 \mathrm{eV}$, see above discussion). Thus, it is possible that these adsorbates will occupy hcp sites (in addition to the weakly favored fcc sites) under a variety of experimental conditions. In our calculations, we observed that these adsorbates induced a radially inward movement of rhodium atoms when placed at hcp sites. This result is consistent with the trend observed for adsorbates whose best site is clearly the hcp site (N, C). The result suggests that the movement of surface atoms does not depend exclusively on the adsorbate in question and that a more general statement about the relationship between adsorbates, site preferences, and surface atom movement can be made. Namely, for atomic adsorbates where adsorption at fcc or hcp sites is energetically competitive, fcc adsorption leads to outward radial movement of the rhodium atoms, and hcp adsorption leads to inward radial movement, irrespective of whether or not the site in question is the most favorable site for the adsorbate.

It is important to note that, while the above trends seem to depend strongly on the adsorption site (fcc or hcp), there does exist a dependence on the identity of the adsorbate. Some molecules do not follow the observed trend; ethylidyne $\left(\mathrm{CCH}_{3}\right)$, for example, binds in hcp sites on $\mathrm{Rh}(111)$ but has been observed experimentally to induce outward radial movement of rhodium surface atoms. ${ }^{41}$

\section{B. Adsorption of molecules and radicals}

The least strongly bound of the molecular adsorbates is dinitrogen with a maximum binding energy of -0.68 $[-0.34] \mathrm{eV}$ at the top site, with the molecule perpendicular to the surface [Table IV and Fig. 3(a)]. We note here that RPBE gives a stable $\mathrm{N}_{2}$ molecular state only for the top site, and thus estimation of a diffusion barrier for $\mathrm{N}_{2}$ on $\mathrm{Rh}(111)$ is meaningless. The RPBE result is in excellent agreement with an estimate of the binding energy based on thermal desorption spectroscopy experiments, $-0.36 \mathrm{eV}$. $^{11}$ The effect of surface relaxation on the binding energy is small, only $-0.06 \mathrm{eV}$. A significant upward shift of $0.20 \AA$ is calculated for the location of the rhodium atom coordinated to the $\mathrm{N}_{2}$ molecule [Table V, Fig. 3(a)].

The methyl radical also favors the top site, binding through the carbon atom with hydrogen atoms pointed toward the neighboring hcp sites [Fig. 3(b)]. A binding energy of $-1.84[-1.49] \mathrm{eV}$ is calculated (Table IV), and a small diffusion barrier, 0.10 [0.20] eV (Table VI), is estimated. The effect of surface relaxation on the binding energy is very small. The $\mathrm{CH}_{3}$-coordinated rhodium atom is shifted upward by $0.12 \AA$ (Table IV).

Another diatomic molecule that prefers the top site is carbon monoxide, bound through the carbon atom and oriented perpendicular to the surface [Fig. 3(c)]. A binding energy of $-2.04[-1.68] \mathrm{eV}$ is calculated (Table IV), and a diffusion barrier of 0.06 [0.14] eV is estimated. Surface relaxation leads to an upward shift of the CO-coordinated rhodium atom by $0.20 \AA$ (Table V). Vibrational spectra of adsorbed carbon monoxide and a core-level photoemission study also indicate a preference for the top site at low coverages. ${ }^{15,16}$ A high resolution photoemission study indicates that at coverages above 0.5 ML the threefold sites become populated, consistent with our finding that the binding
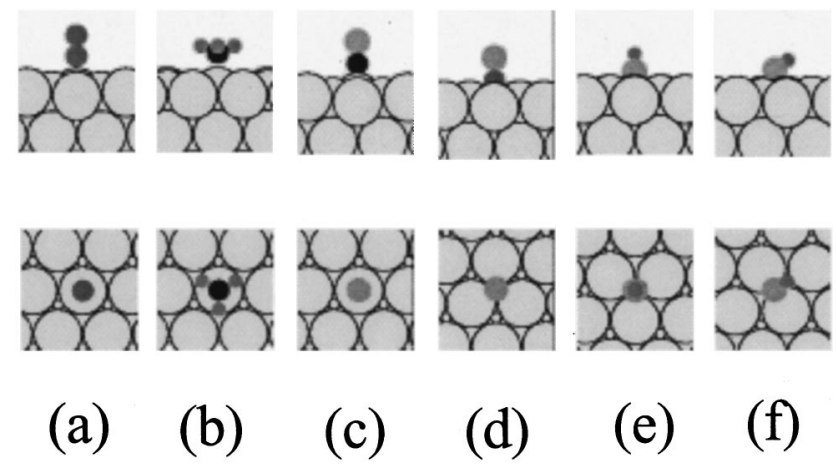

FIG. 3. Preferred binding modes for molecular and radical adsorbates on $\mathrm{Rh}$ (111); (a) $\mathrm{N}_{2}$ top, (b) $\mathrm{CH}_{3}$ top, (c) $\mathrm{CO}$ top, (d) $\mathrm{NO}$ hcp, (e) $\mathrm{OH}$ fcc, (f) $\mathrm{OH}$ bridge tilted. 
TABLE V. Vertical distance between the adsorbate and the plane of three metal atoms defining the corresponding site on relaxed surfaces $\left(d_{\mathrm{A}-\mathrm{Rh}}\right)$, intra-adsorbate $\left(d_{\mathrm{A}-\mathrm{B}}\right)$ bond lengths, displacement of the plane of three metal atoms defining the corresponding sites with respect to the clean, relaxed surface $\left(\Delta z_{\mathrm{Rh}}\right)$, and metal-metal bond length $\left(d_{\mathrm{Rh}-\mathrm{Rh}}\right)$ on the relaxed surface. All configurations are perpendicular to the surface, unless otherwise stated. A schematic representation is given in Fig. 2(b). $d_{\mathrm{Rh}-\mathrm{Rh}}$ on a clean surface $=2.71 \AA$.

\begin{tabular}{ccccc}
\hline \hline Adsorbate & $\begin{array}{c}d_{\mathrm{A}-\mathrm{Rh}} \\
(\AA)\end{array}$ & $\begin{array}{c}d_{\mathrm{A}-\mathrm{B}} \\
(\AA)\end{array}$ & $\begin{array}{c}\Delta z_{\mathrm{Rh}} \\
(\AA)\end{array}$ & $\begin{array}{c}d_{\mathrm{Rh}-\mathrm{Rh}} \\
(\AA)\end{array}$ \\
\hline $\mathrm{N}_{2}$ (top) & 1.91 & 1.14 & 0.20 & $\ldots$ \\
$\mathrm{CH}_{3}$ (top) & 2.09 & 1.10 & 0.12 & $\ldots$ \\
$\mathrm{CO}$ (top) & 1.83 & 1.17 & 0.20 & $\ldots$ \\
$\mathrm{NO}$ (hcp) & 1.30 & 1.22 & 0.09 & 2.68 \\
$\mathrm{OH}$ (fcc) & 1.51 & 0.98 & 0.01 & 2.75 \\
$\mathrm{OH}$ (tilted br) & 1.67 & 0.99 & 0.05 & 2.67 \\
\hline \hline
\end{tabular}

energy for the hcp site is only $0.05[0.14] \mathrm{eV}$ smaller than that of the top site. ${ }^{17}$ The desorption energy for $\mathrm{CO}$ was determined using TPRS and TPD. In both cases the estimated experimental value was $-1.65 \mathrm{eV}$ for $\mathrm{CO}$ coverages below $0.15 \mathrm{ML},{ }^{22,23}$ which is in excellent agreement with our RPBE value $(-1.68 \mathrm{eV})$.

The favored binding site for nitric oxide is hcp [Fig. 3(d)]. This result is in agreement with experimental studies that indicate that at coverages below 0.35 ML, threefold sites are occupied, and at higher coverages, bridge sites become accessible. ${ }^{5,20}$ In the present calculations, the fcc and bridge sites are 0.13 [0.11] and 0.14 [0.10] eV higher in energy, respectively. The preferred orientation of the adsorbed species is perpendicular to the rhodium surface and bound through the nitrogen atom with a binding energy of -2.39 $[-1.84] \mathrm{eV}$ (Table IV). Another DFT calculation for NO on $\mathrm{Rh}(111)$ also yields a preference for the hcp site with a binding energy of $-2.16 \mathrm{eV}^{26}$ There is quite a bit of controversy about the actual experimental value of the desorption energy for NO determined by TPD. Estimated values range from $-1.48 \mathrm{eV}$ (Ref. 22) to $-1.17 \mathrm{eV}^{20}{ }^{20}$ However, at low coverages, such as $0.25 \mathrm{ML}$ where our calculations were performed, dissociation dominates desorption, and therefore accurate experimental estimates for the desorption energy of NO should be very difficult to obtain. Our estimated diffusion barrier for NO is 0.14 [0.11] eV (Table VI). Surface relaxation leads to an increase in binding energy of $0.05 \mathrm{eV}$. The geometric parameters for nitric oxide in the preferred hcp site are given in Table V.

The last species considered in our calculations is the hydroxyl radical. Two structural states are observed that are degenerate in energy. The first state contains the hydroxyl

TABLE VI. Estimates for the diffusion barriers of molecular and radical species on $\operatorname{Rh}(111)$.

\begin{tabular}{ccc}
\hline \hline Adsorbate & $\begin{array}{c}\text { Diffusion barrier (PW91) } \\
(\mathrm{eV})\end{array}$ & $\begin{array}{c}\text { Diffusion barrier (RPBE) } \\
(\mathrm{eV})\end{array}$ \\
\hline $\mathrm{CO}$ & 0.06 & 0.14 \\
$\mathrm{NO}$ & 0.14 & 0.11 \\
$\mathrm{OH}$ & 0.22 & 0.17 \\
$\mathrm{CH}_{3}$ & 0.10 & 0.20 \\
\hline \hline
\end{tabular}

radical bound perpendicular to the surface through oxygen in the fcc site [Fig. 3(e)] with a binding energy of -2.89 $[-2.30] \mathrm{eV}$ (Table IV). The geometric parameters for this site are given in Table V. A previous periodic DFT calculation yielded $-3.34 \mathrm{eV}$ for the binding energy in the fcc site ${ }^{27}$ differences between this finding and our results could be due to the different calculational methods used (FPLAPW versus ultrasoft Vanderbilt pseudopotentials and an unspecified functional versus PW91/RPBE, respectively). The barrier for $\mathrm{OH}$ diffusion on $\mathrm{Rh}(111)$ is estimated to be $\approx 0.22[0.17] \mathrm{eV}$ (Table VI).

The second adsorbed hydroxyl state is a tilted hydroxyl radical bound through oxygen in the bridge site. The hydrogen atom is directed towards the adjacent fcc site at an angle $59.2^{\circ}$ off the vertical [Fig. 3(f)]. The geometric parameters for this configuration are presented in Table V. The binding energy of $\mathrm{OH}$ in this case is $-2.89[-2.33] \mathrm{eV}$ (Table IV), energetically degenerate to the first state. This configuration is similar to an on-top $\mathrm{OH}$ state observed by STM and HREELS on $\mathrm{Pt}(111){ }^{42}$ On that surface, the $\mathrm{OH}$ species were seen to hydrogen bond in six-membered rings.

The above results demonstrate that on-top configurations are favored for $\mathrm{N}_{2}, \mathrm{CO}$, and $\mathrm{CH}_{3}$. These site preferences can be explained by analogy to gas phase bonding trends in an analysis similar to that of Michaelides et al. on $\mathrm{Pt}(111){ }^{43}$ Both $\mathrm{N}_{2}$ and $\mathrm{CO}$ are very stable in the gas phase, and so the lowest possible coordination is expected when these species are adsorbed on a metal surface. This fact is consistent with the calculated on-top geometry. Also, carbon makes four bonds in gas phase molecules, and thus binding to only one metal atom might be expected for the methyl radical. The calculated on-top configuration is consistent with these gas phase coordination arguments. In the cases of $\mathrm{NO}$ and $\mathrm{OH}$, an on-top configuration would be expected from gas phase bonding arguments since nitrogen is trivalent and oxygen is divalent. However, on the rhodium surface, an hcp configuration is preferred for $\mathrm{NO}$ and either fcc or tilted bridge configurations are preferred for $\mathrm{OH}$. Thus, as in the case of atomic adsorbates, it appears that the binding configuration of molecular adsorbates on $\mathrm{Rh}(111)$ cannot be definitively predicted from gas phase bonding considerations.

When we include surface relaxation in our calculations, similar trends are observed in the movement of the rhodium atoms during molecular/radical adsorption as are observed in the case of atomic adsorption. For three of the adsorbates analyzed (CO, NO, and $\mathrm{OH})$, hcp and/or fcc sites demonstrate energetically competitive adsorption properties (i.e., binding energies at the fcc or hcp sites that are within 0.10 $\mathrm{eV}$ of the best binding energies for the adsorbate in question). For NO, the $h c p$ site has the most favorable binding energy (Table IV); a radially inward movement of rhodium atoms is observed in this case, in agreement with the trend established for atomic adsorbates. For $\mathrm{OH}, f c c$ adsorption is favorable. This configuration leads to a radially outward movement of rhodium atoms, again in agreement with the established trend.

A trend similar to the correlation between fcc/hcp site adsorption and the radial movement of surface atoms exists between top site adsorption and vertical movement of the 

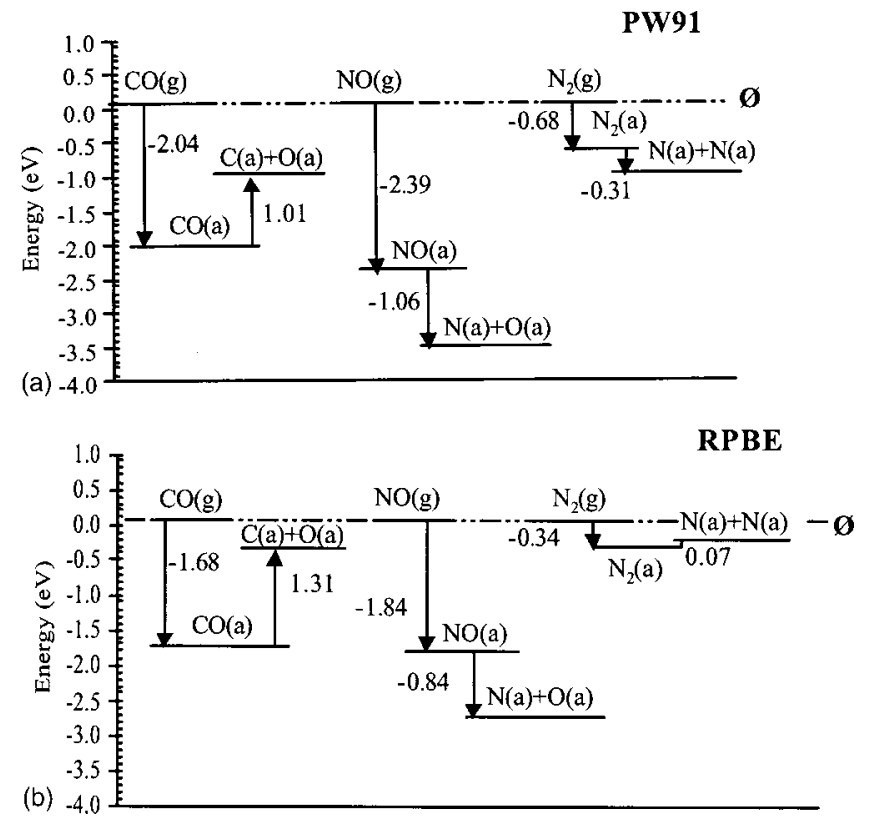

FIG. 4. Thermochemistry of molecular decomposition on the $\mathrm{Rh}(111)$ surface. Reference zero corresponds to gas phase molecules ( $\mathrm{CO}, \mathrm{NO}$, and $\mathrm{N}_{2}$ ) and a $\mathrm{Rh}(111)$ slab at infinite separation. Energetics calculated with the (a) PW91 and (b) RPBE functionals.

surface atoms. Table $\mathrm{V}$ shows that, for all molecules that preferentially adsorb at top sites $\left(\mathrm{N}_{2}, \mathrm{CH}_{3}\right.$, and $\left.\mathrm{CO}\right)$, there is a substantial lifting of the binding $\mathrm{Rh}$ atom out of the surface plane.

For both atomic and molecular adsorbates, a large (multiple tenths of an $\mathrm{eV}$ ) downshift in the magnitude of the binding energy is observed between PW91 and RPBE (Tables I and IV). Interestingly, these downshifts are roughly constant for all configurations for a given adsorbate; this effect might result from differing treatments between the two functionals of the high electron density gradients in gas phase species. The approximately constant downshifts lead to only small changes (generally $<0.1 \mathrm{eV}$ ) in diffusion barriers (Tables III and VI). These diffusion barrier shifts are not of uniform sign (although the shift is consistently downward for atomic adsorbates); unfortunately, the small magnitude of the shifts makes it difficult to conclusively assign a physical interpretation to the sign changes.

\section{Thermochemistry of $\mathrm{CO}, \mathrm{NO}$, and $\mathrm{N}_{2}$ dissociation}

The calculated binding energies and gas phase bond and total energies of several atomic and molecular species were used to construct three simple thermochemical reaction pathways. Figures 4(a) and 4(b) provide the thermochemistry of $\mathrm{CO}, \mathrm{NO}$, and $\mathrm{N}_{2}$ decomposition on $\mathrm{Rh}(111)$ based on the PW91 and RPBE functionals, respectively.

It is seen that there is a $-1.03[-0.37] \mathrm{eV}$ gain in energy due to adsorption followed by decomposition of $\mathrm{CO}$ although the dissociation of adsorbed $\mathrm{CO}$ is endothermic with respect to the molecular $\mathrm{CO}$ adsorbed state. Further, a recent DFT study shows that the barrier for $\mathrm{CO}$ dissociation on $\mathrm{Rh}(111)$ is $1.48 \mathrm{eV}$ (RPBE) above the gas phase zero. ${ }^{30}$ This result, combined with the present calculations, indicates that the barrier for $\mathrm{CO}$ desorption is lower than the activation barrier for dissociation. These findings are in good agreement with the results of a TPD study on $\mathrm{Rh}(111)$; that study demonstrated that $\mathrm{CO}$ decomposition is not observed on $\mathrm{Rh}(111)$ under experimental conditions $\left(P<10^{-5}\right.$ Torr CO, $\left.T<600 \mathrm{~K}\right) .^{15}$

The second thermochemical pathway investigated is the adsorption and decomposition of NO (Fig. 4). The net energy gain from dissociative adsorption of $\mathrm{NO}$ on $\mathrm{Rh}(111)$ is $-3.45[-2.68] \mathrm{eV}$. Clearly the dissociation of adsorbed NO is exothermic. TPD experiments indicate that, in ultrahigh vacuum (UHV) conditions, for NO coverages below 0.25 ML, NO dissociates at $\sim 300 \mathrm{~K}$; it desorbs at $\sim 380 \mathrm{~K}$ from $\mathrm{Rh}(111)$ only after enough atomic $\mathrm{N}$ and $\mathrm{O}$ has been produced by the dissociation process so that surface sites are partially blocked and further dissociation becomes impossible. ${ }^{20}$ Thus, the activation energy for breaking the $\mathrm{N}-\mathrm{O}$ bond is smaller than the binding energy of $\mathrm{NO}$ on $\mathrm{Rh}(111)$. Our DFT calculations show that the dissociative adsorption process is highly thermodynamically favorable, consistent with the experimental data.

The last bond breaking reaction investigated is that of molecular nitrogen. Adsorption and decomposition of $\mathrm{N}_{2}$ yields a total energy gain of $-0.99[-0.27] \mathrm{eV}$ (Fig. 4) on $\mathrm{Rh}(111)$. Although the RPBE results indicate that the dissociation of adsorbed $\mathrm{N}_{2}$ on $\mathrm{Rh}(111)$ is quasithermoneutral, kinetic limitations prohibit this reaction from occurring. Preliminary calculations indicate that the barrier for $\mathrm{N}_{2}$ dissociation is, indeed, much larger than the binding energy of $\mathrm{N}_{2}$ on $\mathrm{Rh}(111)$, suggesting that $\mathrm{N}_{2}$ would desorb from $\mathrm{Rh}(111)$ instead of dissociating on that surface.

\section{CONCLUSIONS}

The chemisorption of various atomic $(\mathrm{H}, \mathrm{O}, \mathrm{N}, \mathrm{S}$, and C), molecular $\left(\mathrm{N}_{2}, \mathrm{CO}, \mathrm{NO}\right)$, and radical $\left(\mathrm{CH}_{3}, \mathrm{OH}\right)$ species on $\mathrm{Rh}(111)$ at $0.25 \mathrm{ML}$ coverage has been studied via periodic, self-consistent DFT calculations. The relative binding energies for the investigated species are $\mathrm{N}_{2}<\mathrm{CH}_{3}<\mathrm{CO}$ $<\mathrm{NO}<\mathrm{H}<\mathrm{OH}<\mathrm{O}<\mathrm{N}<\mathrm{S}<\mathrm{C}$ (PW91), from the least to the most strongly bound species. In all cases where experimental data exist, the calculated RPBE binding energies are found to be in excellent agreement with the available experimental values.

It is generally observed that the preferred binding sites for the atomic adsorbates are threefold hollow sites. For the molecular adsorbates, the top site is preferred for most cases. However, this site-preference does not hold for all of the molecular adsorbates. Nitric oxide prefers hcp sites while hydroxyl can be found in either fcc or bridge sites.

Molecular species generally bind perpendicular to the $\mathrm{Rh}(111)$ surface. However, for the hydroxyl radical, a tilted chemisorbed state exists that is energetically degenerate with the perpendicular fcc configuration. This state consists of the $\mathrm{OH}$ bound through oxygen in the bridge site with hydrogen directed towards a neighboring fcc site.

Surface relaxation of $\mathrm{Rh}(111)$ was found to be of secondary importance for the binding energy of the adsorbates studied. However, for molecular adsorbates bound to the top 
site, a noticeable vertical upshift of the binding rhodium atom was found.

All atomic adsorbates, except for $\mathrm{H}$, have a significant diffusion barrier, between 0.4 and $0.6 \mathrm{eV}$ (RPBE) on $\mathrm{Rh}(111)$. Atomic $\mathrm{H}$ and molecular/radical adsorbates appear to be much more mobile on $\mathrm{Rh}(111)$, with an estimated diffusion barrier between 0.1 and $0.2 \mathrm{eV}$ (RPBE).

The calculated thermochemistry for the decomposition of adsorbed $\mathrm{CO}, \mathrm{NO}$, and $\mathrm{N}_{2}$ on $\mathrm{Rh}(111)$ varies. In RPBE it is clearly exothermic for $\mathrm{NO}$, endothermic for $\mathrm{CO}$, and almost thermoneutral for $\mathrm{N}_{2}$.

\section{ACKNOWLEDGMENTS}

One of the authors (J.R.) acknowledges a Holstrom Environmental Fellowship for Undergraduate Research at the University of Wisconsin-Madison. J.G. acknowledges financial support from a National Science Foundation predoctoral fellowship. M.M. acknowledges partial financial support from a NSF-CAREER Award (CTS-0134561) and from a 3M Faculty Award. Part of the calculations was performed at the Department of Energy's National Energy Research Scientific Computing Center. Authors acknowledge partial support from NSF cooperative agreement ACI-9619020 through computing resources provided by the National Partnership for Advanced Computational Infrastructure (NPACI). L.B.H. and J.K.N. have been supported by the Danish National Research Councils.

${ }^{1}$ C. N. Satterfield, Heterogeneous Catalysis in Industrial Practice, 2nd ed. (Krieger, Malabar, 1996).

${ }^{2}$ S. Schwegmann, H. Over, V. De Renzi, and G. Ertl, Surf. Sci. 375, 91 (1997).

${ }^{3}$ A. J. Jaworowski, A. Beutler, F. Strisland, R. Nyholm, B. Setlik, D. Heskett, and J. N. Andersen, Surf. Sci. 431, 33 (1999).

${ }^{4}$ K. D. Gibson, M. Viste, E. Sanchez, and S. J. Sibener, J. Chem. Phys. 112, 2470 (2000).

${ }^{5}$ H. Xu and K. Y. S. Ng, Surf. Sci. 365, 779 (1996).

${ }^{6}$ G. Comelli, V. R. Dhanak, M. Kiskinova, K. C. Prince, and R. Rosei, Surf. Sci. Rep. 32, 165 (1998).

${ }^{7}$ J. A. Rodriguez, S. Chaturvedi, and M. Kuhn, J. Chem. Phys. 108, 3064 (1998).

${ }^{8}$ H. A. Yoon, M. Salmeron, and G. A. Somorjai, Surf. Sci. 395, 268 (1998).

${ }^{9}$ H. Yanagita, H. Fujioka, T. Aruga, N. Takagi, and N. Nishijima, Surf. Sci. 441, 507 (1999).

${ }^{10}$ S. S. Mann, T. Seto, C. J. Barnes, and D. A. King, Surf. Sci. 261, 155 (1991).
${ }^{11}$ H. A. C. M. Hendrickx, A. Hoek, and B. E. Nieuwenhuys, Surf. Sci. 135, 81 (1983).

${ }^{12}$ D. G. Castner, B. A. Sexton, and G. A. Somorjai, Surf. Sci. 71, 519 (1978)

${ }^{13}$ I. Zasada, M. A. Van Hove, and G. A. Somorjai, Surf. Sci. 418, L89 (1998).

${ }^{14}$ T. W. Root, G. B. Fisher, and L. D. Schmidt, J. Chem. Phys. 85, 4679 (1986).

${ }^{15}$ L. H. Dubois and G. A. Somorjai, Surf. Sci. 91, 514 (1980).

${ }^{16}$ A. Beutler, E. Lundgren, R. Nyholm, J. N. Andersen, B. J. Setlik, and D. Heskett, Surf. Sci. 396, 117 (1998).

${ }^{17}$ A. Beutler, E. Lundgren, R. Nyholm, J. N. Andersen, B. Setlik, and D. Heskett, Surf. Sci. 371, 381 (1997)

${ }^{18}$ P. Cernota, K. Rider, H. A. Yoon, M. Salmeron, and G. Somorjai, Surf. Sci. 445, 249 (2000).

${ }^{19}$ J. Kiss, A. Kis, and F. Solymosi, Surf. Sci. 454-456, 273 (2000).

${ }^{20}$ H. J. Borg, J. F. C.-J. M. Reijerse, R. A. van Santen, and J. W. Niemantsverdriet, J. Chem. Phys. 101, 10052 (1994).

${ }^{21}$ M. J. P. Hopstaken and J. W. Niemantsverdriet, J. Chem. Phys. 113, 5457 (2000).

${ }^{22}$ V. P. Zhdanov and B. Kasemo, Catal. Lett. 40, 197 (1996).

${ }^{23}$ M. J. P. Hopstaken, W. J. H. v. Gennip, and J. W. Niemantsverdriet, Surf. Sci. 433-435, 69 (1999).

${ }^{24}$ D. N. Belton, C. L. DiMaggio, and K. Y. S. Ng, J. Catal. 144, 273 (1993).

${ }^{25}$ M. V. Ganduglia-Pirovano and M. Scheffler, Phys. Rev. B 59, 15533 (1999).

${ }^{26}$ D. Loffreda, D. Simon, and P. Sautet, J. Chem. Phys. 108, 6447 (1998).

${ }^{27}$ S. Wilke, V. Natoli, and M. H. Cohen, J. Chem. Phys. 112, 9986 (2000).

${ }^{28}$ C. J. Zhang, P. Hu, and M. H. Lee, Surf. Sci. 432, 305 (1999).

${ }^{29}$ Z.-P. Liu and P. Hu, J. Chem. Phys. 114, 8244 (2001).

${ }^{30}$ M. Mavrikakis, M. Bäumer, H. J. Freund, and J. K. Nørskov, Catal. Lett. 81, 153 (2002).

${ }^{31}$ B. Hammer, L. B. Hansen, and J. K. Nørskov, Phys. Rev. B 59, 7413 (1999).

${ }^{32}$ J. Neugebauer and M. Scheffler, Phys. Rev. B 46, 16067 (1992).

${ }^{33}$ D. Vanderbilt, Phys. Rev. B 41, 7892 (1990).

${ }^{34}$ J. P. Perdew, J. A. Chevary, S. H. Vosko, K. A. Jackson, M. R. Pederson, D. J. Singh, and C. Fiolhais, Phys. Rev. B 46, 6671 (1992).

${ }^{35}$ J. A. White and D. M. Bird, Phys. Rev. B 50, 4954 (1994).

${ }^{36}$ G. Kresse and J. Furthmuller, Comput. Mater. Sci. 6, 15 (1996).

${ }^{37}$ CRC Handbook of Chemistry and Physics, 76th ed., edited by D. R. Lide (CRC Press, New York, 1996).

${ }^{38}$ J. T. Yates, P. A. Thiel, and W. H. Weinberg, Surf. Sci. 84, 427 (1979).

${ }^{39}$ W. A. Brown, R. Kose, and D. A. King, Chem. Rev. 98, 797 (1998).

${ }^{40}$ P. J. Feibelman, B. Hammer, J. K. Nørskov, F. Wagner, M. Scheffler, R. Stumpf, R. Watwe, and J. Dumesic, J. Phys. Chem. B 105, 4018 (2001).

${ }^{41}$ G. A. Somorjai, Introduction to Surface Chemistry and Catalysis (Wiley, New York, 1994).

${ }^{42}$ K. Bedurftig, S. Volkening, Y. Wang, J. Wintterlin, K. Jacobi, and G. Ertl, J. Chem. Phys. 111, 11147 (1999).

${ }^{43}$ A. Michaelides and P. Hu, J. Am. Chem. Soc. 122, 9866 (2000). 